\title{
Impact of a defunctioning ileostomy and time to stoma closure on bowel function after low anterior resection for rectal cancer: a systematic review and meta-analysis
}

\author{
I. Vogel ${ }^{1,2}$ (I) N. Reeves ${ }^{2} \cdot$ P. J. Tanis ${ }^{1} \cdot$ W. A. Bemelman ${ }^{1} \cdot$ J. Torkington ${ }^{2} \cdot$ R. Hompes ${ }^{1} \cdot$ J. A. Cornish ${ }^{2}$
}

Received: 24 October 2020 / Accepted: 10 March 2021 / Published online: 1 April 2021

(c) Crown 2021

\begin{abstract}
Background Impaired bowel function after low anterior resection (LAR) for rectal cancer is a frequent problem with a major impact on quality of life. The aim of this study was to assess the impact of a defunctioning ileostomy, and time to ileostomy closure on bowel function after LAR for rectal cancer.

Methods We performed a systematic review based on the preferred reporting items for systematic reviews and meta-analyses (PRISMA) statement. Comprehensive literature searches were conducted using PubMed, Embase and Cochrane databases for articles published from 1989 up to August 2019. Analysis was performed using Review Manager (version 5.3) using a random-effects model.

Results The search yielded 11 studies (1400 patients) that reported on functional outcome after LAR with at least 1 year follow-up, except for one study. Five scales were used: the Low Anterior Resection Syndrome (LARS) score, the Wexner score, the Memorial Sloan Kettering Cancer Centre Bowel Function Instrument, the Fecal Incontinence Quality of Life scale, and the Hallbook questionnaire. Based on seven studies, major LARS occurred more often in the ileostomy group (OR 2.84, 95\% CI, 1.70-4.75, $p<0.0001: I^{2}=60 \%, X^{2}=0.02$ ). Based on six studies, a longer time to stoma closure increased the risk of major LARS with a mean difference in time to closure of 2.39 months (95\% CI, 1.28-3.51, $p<0.0001: I^{2}=21 \%, X^{2}=0.28$ ) in the major vs. no LARS group. Other scoring systems could not be pooled, but presence of an ileostomy predicted poorer bowel function except with the Hallbook questionnaire.

Conclusions The risk of developing major LARS seems higher with a defunctioning ileostomy. A prolonged time to ileostomy closure seems to reinforce the negative effect on bowel function; therefore, early reversal should be an important part of the patient pathway.
\end{abstract}

Keywords Low anterior resection · Rectal cancer · Ileostomy · Bowel function · Low anterior resection syndrome

\section{Introduction}

Low anterior resection (LAR) with total mesorectal excision is the gold standard surgical procedure for patients with resectable primary rectal cancer [1]. When intestinal continuity with a colorectal or coloanal anastomosis is restored,

R. Hompes and J. A. Cornish shared senior authorship.

$\triangle$ I. Vogel

i.vogel@amsterdamumc.nl

1 Department of Surgery, Amsterdam UMC, University of Amsterdam, Amsterdam, The Netherlands

2 Department of Colorectal Surgery, University Hospital of Wales, Cardiff, UK the procedure is often routinely combined with a temporary stoma, to mitigate potential severe sequelae of an anastomotic leak [2]. In general patients have a defunctioning stoma for 3-6 months, however it is not unusual that this period extends beyond 12 months prolonging the negative impact that the presence of an ileostomy has on the patient's physical, psychological and social wellbeing [3-5].

In patients with a functioning anastomosis, impaired bowel function is a frequent problem with a major impact on quality of life $[6,7]$. The symptoms are often referred to as low anterior resection syndrome (LARS), and include; urgency, difficulty emptying and incontinence [8]. A recent meta-analysis found a combined prevalence of major LARS of $41 \%$ at least 1 year after stoma closure, when further improvement of the symptoms is unlikely [7]. Radiotherapy 
and the level of the anastomosis are known to have a negative impact on major LARS, but the presence of an ileostomy and prolonged time to ileostomy closure are mentioned as possible risk factors [9]. However, the literature is inconsistent, with few studies reporting on functional outcome after LAR with and without ileostomy.

Therefore, we performed a systematic review and metaanalysis of the current literature to evaluate the impact of a defunctioning loop ileostomy on bowel function after LAR for rectal cancer. A secondary aim was to assess whether time to stoma closure after the index operation had an effect on bowel function.

\section{Materials and methods}

\section{Study selection}

This systematic review and meta-analysis was conducted in line with the Preferred Reporting Items for Systematic Reviews and Meta-Analysis (PRISMA) guidelines (available at. Prisma-statement.org) [10]. Comprehensive literature searches were conducted using PubMed, Embase and Cochrane databases for articles published from 1989 up to August 2019. The full search strategy is displayed in the Appendix: Table S1-3.

Studies were considered for inclusion if they met the following criteria: (1) patients diagnosed with rectal cancer within $15 \mathrm{~cm}$ from the anal verge; (2) patients had LAR with a colorectal or coloanal anastomosis with or without defunctioning ileostomy; (3) assessment of bowel function following LAR with a validated tool; (4) studies were cohort, case matched studies or randomized clinical trials. The exclusion criteria were as follows: (1) reviews, letters, expert opinions, commentaries, case series or case reports; (2) language other than English; (3) lack of the sufficient data or outcomes of interest; (4) duplicate studies; (4) intersphincteric resection.

Two reviewers (IV and NR) independently reviewed titles and abstracts, followed by full text revision. Additionally, the references of relevant studies were hand-searched. Authors of relevant conference abstracts or of studies with missing data were contacted to request more details, 3 authors responded and were able to provide their data sets which made inclusion in our meta-analysis possible [1, 11, 12]. Disagreements were resolved by consensus discussion between the two reviewers (IV and NR).

\section{Data extraction and quality assessment}

Data were extracted independently by two authors (IV and NR) and included the following variables: year of publication, country, study design, number of patients, characteristics of included patients, neoadjuvant treatment, distance of the tumor from the anal verge, proportion of patients undergoing partial mesorectal excision, percentage of anastomotic leaks, number of ileostomies, time to ileostomy closure, length of follow -up and reported scoring systems to assess bowel function.

The primary outcome was bowel function after LAR for rectal cancer in patients with and without an ileostomy and also LARS as a function of time of ileostomy closure.

Risk of bias was assessed using the Newcastle-Ottowa scale for cohort studies and Jadad scoring system for randomized controlled trials $[13,14]$. When randomized groups of the randomized controlled trials (RCTs) were not used as described in the RCT, the Newcastle Ottawa quality assessment was used. Two of the authors (IV and NR) performed the quality assessment, and conflicts were discussed to achieve consensus.

\section{Statistical analysis}

Heterogeneity was assessed using the $I^{2}$ and $X^{2}$ statistics, the data were considered significant if the $p$ value $\left(X^{2}\right)$ was $<0.1$ with low, moderate, and high for $I^{2}$ values of $25 \%, 50 \%$, and $75 \%$. Analysis was performed using Review Manager (RevMan, version 5.3. Copenhagen: The Nordic Centre, the Cochrane Collaboration, 20) with a random-effects model. The Mantel-Haenzel method was used to calculate the odds ratio and Inverse Variance method was used to calculate the mean difference, both illustrated in forest plots with a 95\% confidence interval. When a median time to closure was presented with an interquartile range the our method was used to covert to mean time to closure with a standard deviation.

\section{Results}

In total, 1627 articles were screened on title and abstract (Appendix: Table S1-3). Fifty-nine studies were remained for a full text review. After exclusion of 48 studies, a total of 11 studies with 1400 patients, were included in our systematic review (Fig. 1). Seven studies were cohort studies and four studies were secondary analysis of randomized controlled trials. One randomized controlled trial was used as a cohort study [15]. The assessment for methodological quality and risk of bias is listed in the Appendix: Table S4.

\section{Patient characteristics}

Baseline characteristics of the studies are listed in Table 1. All patients had LAR for rectal cancer with a colorectal or coloanal anastomosis. Ten studies mentioned the 

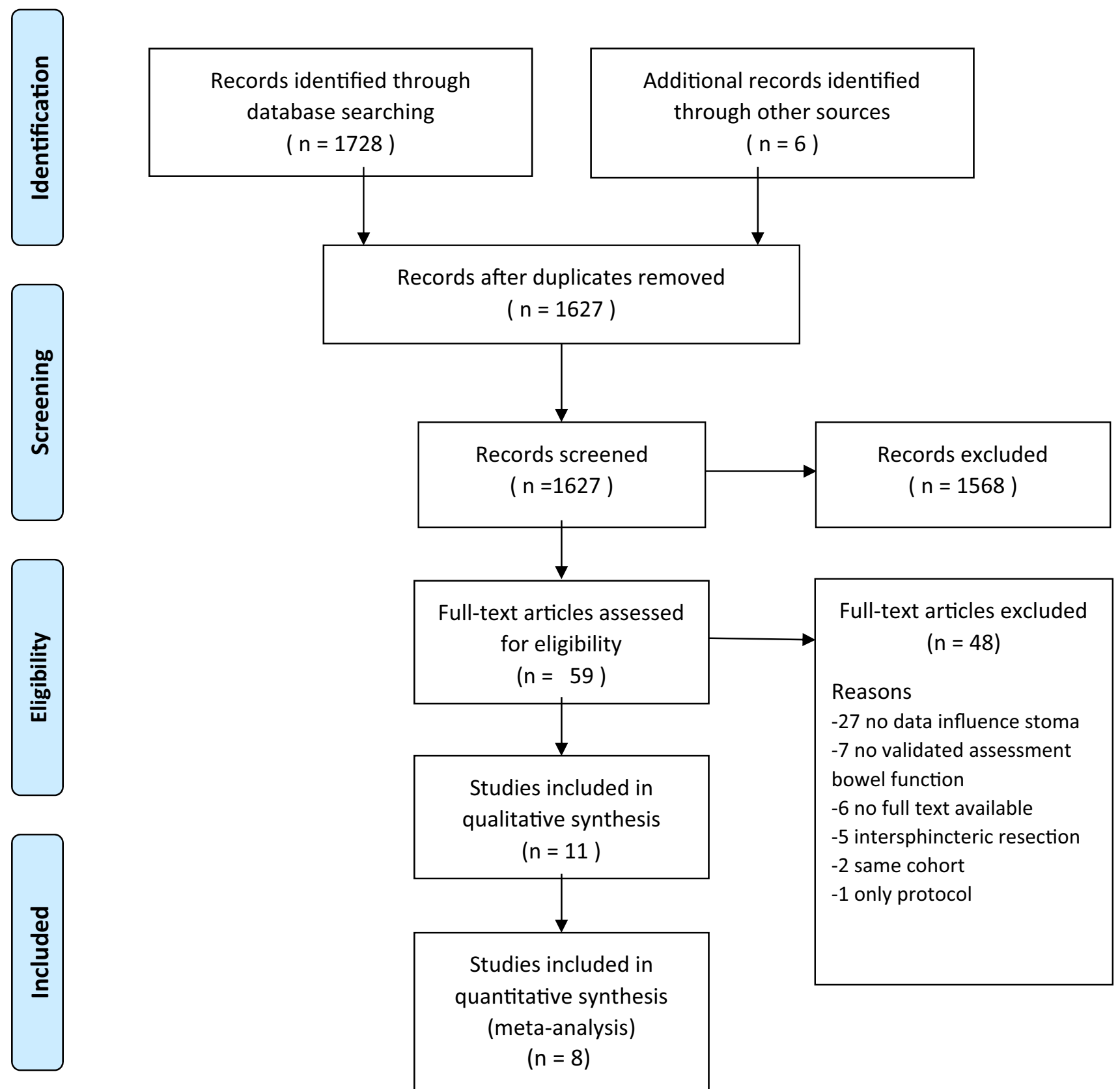

Fig. 1 PRISMA flow diagram

proportion of patients receiving neoadjuvant radiotherapy, which ranged from $26 \%$ to $97 \%$ [1, 11, 12, 15-21]. The majority were mid and proximal rectal cancers $(5-15 \mathrm{~cm}$ from the anal verge), with varying proportions of distal tumors $(<5 \mathrm{~cm})$ ranging from 0 to $37 \%$ (Table 1). Five studies reported the number of patients that underwent a total or partial mesorectal excision, 3 studies reported $100 \%$ total mesorectal excision (TME), partial mesorectal excision was mentioned in 2 studies, and constituted $27 \%$ and $50 \%$ of their study population, respectively $[17,19]$.
In total, $61.2 \%$ of patients $(N=857,29 \%$ to $100 \%$ in the included studies). received an ileostomy, In one study, by Keane et al., $100 \%$ of the patients received an ileostomy [11]. Eight studies reported on acute anastomotic leaks with an incidence of $0-12.7 \%[1,11,12,15,17,19-21]$.

\section{Functional outcomes}

Five different scales were used to report on bowel function: the LARS score, Wexner score, bowel function questionnaire by Hallbook, Memorial Sloan Kettering Cancer Centre 


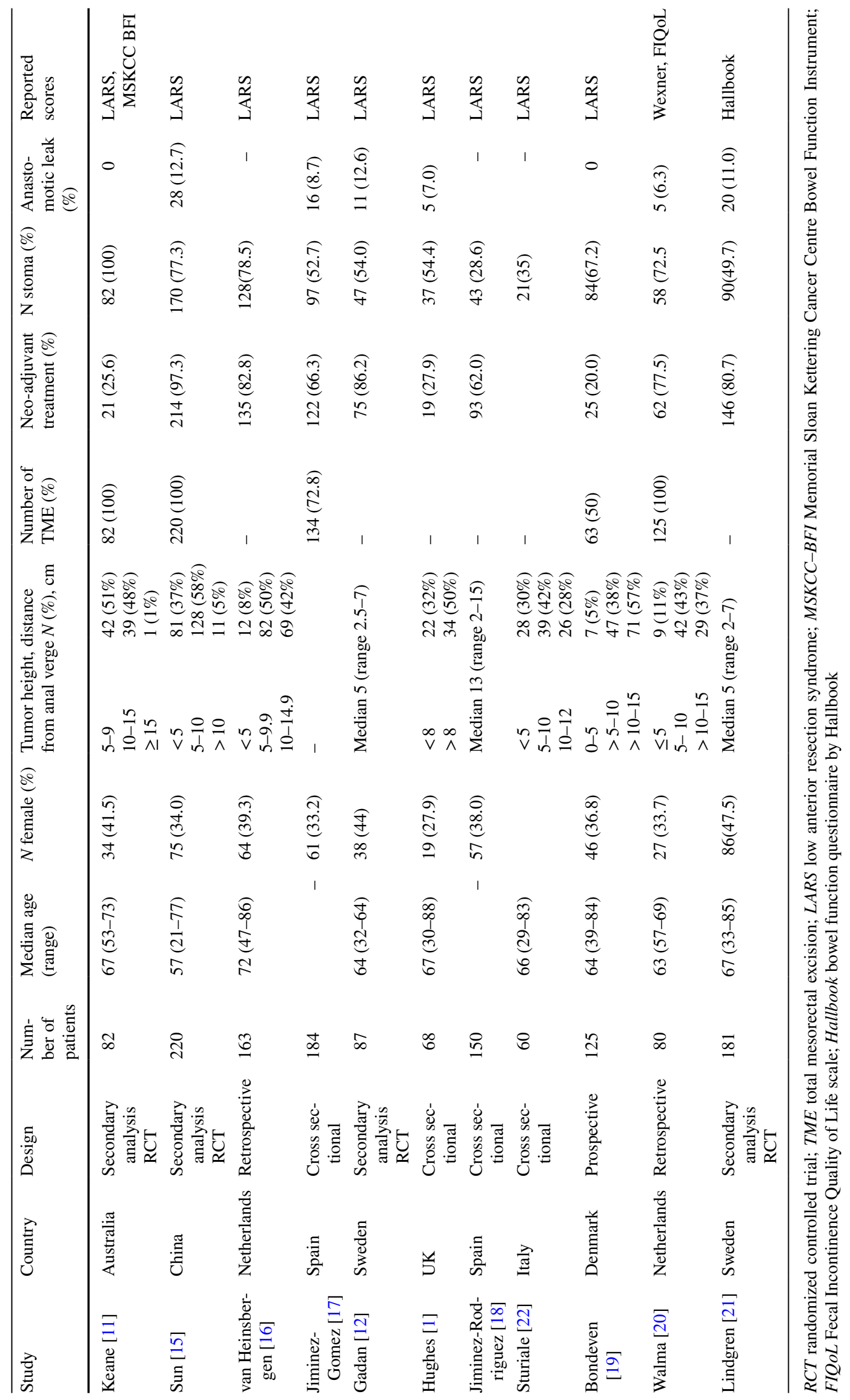


Table 2 LARS score after LAR for rectal cancer

\begin{tabular}{lllcc}
\hline Author & No LARS (\%) & Minor LARS (\%) & Major LARS (\%) & $\begin{array}{l}\text { Time from surgery to } \\
\text { LARS median months } \\
\text { (range) }\end{array}$ \\
\hline Keane [11] & $16(20)$ & $12(15)$ & $54(65.9)$ & $49(24-77)^{*}$ \\
Sun [15] & $27(12.3)$ & $74(33.6)$ & $119(54.1)$ & $40.2(23-87)$ \\
van Heinsbergen [16] & $47(28.8)$ & $29(47.3)$ & $87(53.4)$ & $62.4(28-100)$ \\
Jiminez-Gomez [17] & $44(23.9)$ & $36(19.6)$ & $104(56.2)$ & $45.7(31-64)^{*}$ \\
Gadan [12] & $24(27.5)$ & $17(19.5)$ & $46(52.9)$ & $141.8(117-177)$ \\
Hughes [1] & $18(26)$ & $12(18)$ & $38(58)$ & $8.2(0.6-55)$ \\
Jimiez-Rodriquez [18] & $82(54.7)$ & $26(17.3$ & $42(28.0)$ & $36(12-60)$ \\
Sturiale [22] & $32(53.3)$ & $15(25)$ & $13(21.7)$ & $164.4(131-216)$ \\
Bondeven [19] & $48(38)$ & $30(24)$ & $47(38)$ & $18(12-24)$ \\
Total & $338(29.7)$ & $251(22.0)$ & $550(48.3)$ & \\
\hline
\end{tabular}

LARS low anterior resection syndrome

*Presented with interquartile range instead of range
Bowel Function Instrument (MSKCC-BFI) and Fecal Incontinence Quality of Life (FIQoL) scale. Nine studies used the LARS score and the remaining scores were used by only 1 study each, specified in Table 1 . An explanation of the different scoring systems is in the Appendix: Table S5 [23-27]. Median time to stoma closure was reported in 8 studies and varied from under 3.4 to 19 months (Table 3) [1, 11, 12, 15-18, 21]. Six studies reported the number of patients that could not be included in the assessment of bowel function because of a permanent stoma, ranging from $6.3 \%$ to $27.7 \%$ of the total number of patients that were assessed for eligibility $[11,12,18,20-22]$. The number of patients not eligible for inclusion because of death was reported in five studies and ranged from $5.9 \%$ to $49.6 \%$ [11, 12, 18, 20, 22] .

\section{LARS score}

Nine studies reported on LARS score in 1139 patients with a median follow-up time of 54.3 months (range 8.2-164.4 months) $[1,11,12,15-19,22]$. With the exception of Hughes et al. [1], follow-up time was more than 1 year in all studies. Overall $48.3 \%$ of the patients reported major LARS $(n=550)$, while minor LARS or no LARS were reported in $22.0 \%$ and $29.7 \%$ of the patients respectively (Table 2). In six studies, major LARS was

Table 3 Reported time to ileostomy closure and functional score

\begin{tabular}{|c|c|c|c|c|c|c|}
\hline Study & Reported scores & $\begin{array}{l}\text { Dropout due to } \\
\text { permanent stoma } \\
(\%)^{*}\end{array}$ & $\begin{array}{l}\text { Dropout due } \\
\text { to death }(\%)^{*}\end{array}$ & $\begin{array}{l}N \text { patients } \\
\text { with stoma } \\
(\%)\end{array}$ & $\begin{array}{l}\text { Median months } \\
\text { to stoma closure } \\
\text { (range) }\end{array}$ & $\begin{array}{l}\text { Follow-up time } \\
\text { months (range) }\end{array}$ \\
\hline Keane [11] & LARS, MSKCC BFI & $7(6.3)$ & $12(10.7)$ & $82(100)$ & $3.4(0.3-12.2)$ & $49(24-77)^{* *}$ \\
\hline Sun [15] & LARS & - & - & $170(77.3)$ & $5.3(0.6-22.9)$ & $40.2(23-87)$ \\
\hline van Heinsbergen [16] & LARS & - & - & $128(78.5)$ & $4.5(0.2-16.3)$ & $62.4(28-100)$ \\
\hline Jiminez-Gomez [17] & LARS & - & - & $97(52.7)$ & & $45.7(31-64)^{* *}$ \\
\hline Gadan [12] & LARS & $27(11.5)$ & 109 (27.7) & $47(54.0)$ & $5.1(0.4-19.6)$ & $141.8(117-177)$ \\
\hline Hughes [1] & LARS & & & $37(54.4)$ & $7.0(1.6-11.9)$ & $8.2(0.6-55)$ \\
\hline Jiminez-Rodriguez [18] & LARS & 109 (27.7) & 105 (26.6) & $43(28.6)$ & $15(3-31)$ & $36(12-60)$ \\
\hline Sturiale [22] & LARS & $39(10.3)$ & $177(46.6)$ & $21(35)$ & - & $164.4(131-216)$ \\
\hline Bondeven [19] & LARS & - & - & $84(67.2)$ & - & $18(12-24)$ \\
\hline Walma [20] & Wexner, FIQoL & $21(8.7)$ & $14(5.9)$ & $58(72.5$ & $19(10-29)$ & $19(10-29) * *$ \\
\hline Lindgren [21] & Hallbook & $33(24.8)$ & - & $90(49.7)$ & $6(1-21)$ & $12(7-24)$ \\
\hline
\end{tabular}

LARS low anterior resection syndrome; MSKCC-BFI Memorial Sloan Kettering Cancer Centre Bowel Function Instrument; FIQoL Fecal Incontinence Quality of Life scale; Hallbook bowel function questionnaire by Hallbook

*Percentage of total number of patient in cohort that received an anterior resection for rectal cancer and were assessed for eligibility bowel function questionnaire

** Median presented with interquartile range instead of range 
significantly more frequent in the group of patients that received an ileostomy in univariate analysis [12, 15-19]. An ileostomy was also associated with increased risk of major LARS in multivariate analysis after correction for neoadjuvant therapy and tumor height as confounding factors in two studies $[15,16]$. Higher rates of major LARS in defunctioned patients were also found in the other studies, but the results were not statistically significant $[1,22]$. In the study by Keane et al., all patients were defunctioned, which made the assessment of the impact of a stoma impossible.

A total of 894 patients from seven studies were included for the pooled analysis after excluding van Heinsbergen et al. (no separate data on presence of stoma available for rectal cancer population); major LARS occurred significantly more often in the ileostomy group compared to the non defunctioned patients with an OR of 2.84 and moderate levels of heterogeneity between the included studies (95\% CI, 1.70-4.75 $p<0.0001: I^{2}=60 \%, X^{2}=0.02$ ), Fig. 2 $[1,12,15,17-19,22]$.

\section{Impact of timing of stoma closure}

Six studies evaluated the influence of timing of ileostomy closure on LARS score (Table 3) [1, 11, 12, 15, 17, 18].
Hughes et al. showed in multivariate analysis that ileostomy reversal within 6 months after initial surgery was protective against major LARS (OR 0.2, 95\% CI, 0.1-0.3, $p<0.01$ ) and that reversal after 1 year was associated with increased risk of major LARS (OR 3.7, CI 95\%, 1.1-13.1, $p=0.03$ ) [1]. An increased risk of major LARS after longer time to ileostomy closure was also found in 4 other studies, but without a statistically significant difference $[11,12,15,17]$. Only one study reported no influence of timing of stoma closure on LARS score; in this trial there was only a 2 week difference in the time to closure in the major LARS vs no LARS group and the mean time was 15.1 months vs 15.6 months respectively [18].

Six studies, on 719 patients, were included in pooled analysis on the influence of timing of ileostomy closure on prevalence of major LARS. Mean time to ileostomy closure ranged from 2.4 to 15.6 months with a mean difference of 2.39 months (95\% CI, 1.28-3.51, $p<0.0001: I^{2}=21 \%$, $\left.X^{2}=0.28\right)$ in the major vs. no LARS group with low levels of heterogeneity between the individual studies $[1,11,12$, 15, 17, 18] (Fig. 3). Patients with major LARS had an average time of ileostomy closure 10 weeks later than those with no LARS.

\begin{tabular}{|c|c|c|c|c|c|}
\hline \multirow[b]{2}{*}{ Study or Subgroup } & \multicolumn{2}{|c|}{ lleostomy } & \multicolumn{2}{|c|}{ No ileostomy } & \multirow[b]{2}{*}{ Weight } \\
\hline & Events & Total & Events & Total & \\
\hline Bondeven 2015 & 44 & 84 & 3 & 41 & $10.1 \%$ \\
\hline Jiminez-Rodriquez 2016 & 19 & 43 & 23 & 107 & $16.2 \%$ \\
\hline Jiminez-Gomez 2017 & 65 & 97 & 39 & 87 & $18.7 \%$ \\
\hline Gadan 2017 & 24 & 41 & 23 & 46 & $14.9 \%$ \\
\hline Sturiale 2017 & 6 & 21 & 7 & 39 & $10.1 \%$ \\
\hline Hughes 2017 & 22 & 37 & 16 & 31 & $13.3 \%$ \\
\hline Sun 2019 & 107 & 119 & 63 & 101 & $16.8 \%$ \\
\hline Total $(95 \% \mathrm{Cl})$ & & 442 & & 452 & $100.0 \%$ \\
\hline Total events & 287 & & 174 & & \\
\hline
\end{tabular}

Odds Ratio Odds Ratio

Random, 95\% Cl Year $13.93[3.99,48.68] 2015$ $2.89[1.35,6.17] 2016$

$2.50[1.37,4.55] 2017$

$1.41[0.60,3.30] 2017$

$1.83[0.52,6.39] 2017$

$1.38[0.53,3.60] 2017$

$5.38[2.62,11.05] 2019$

$2.84[1.70,4.75]$

Event is defined as major LARS

LARS = low anterior resection syndrome

Fig. 2 Prevalence of major LARS in ileostomy and no ileostomy group. Event is defined as major LARS. LARS low anterior resection syndrome

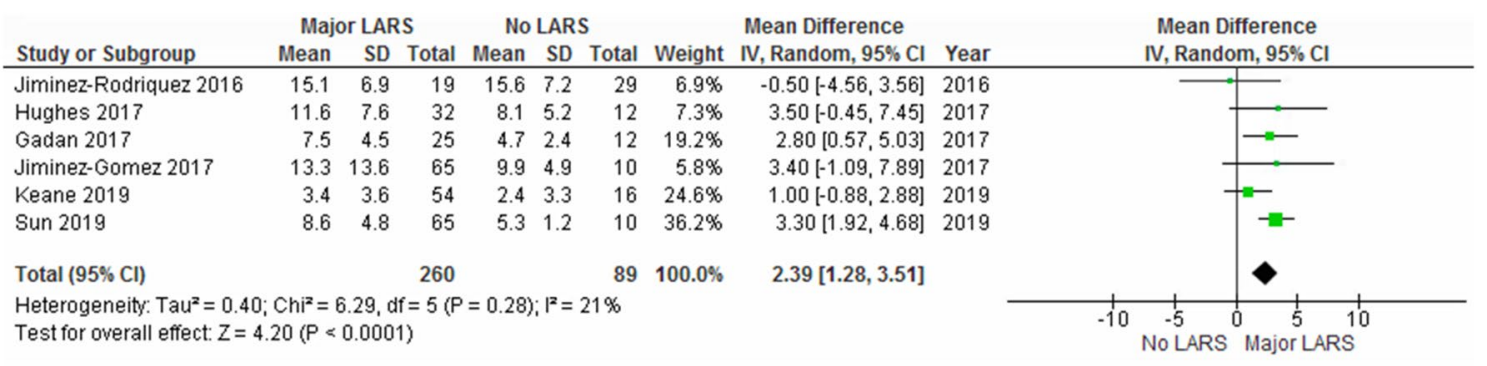

LARS= low anterior resection syndrome $; \mathrm{SD}=$ standard deviation

Fig. 3 Time to stoma closure major LARS vs no LARS. LARS low anterior resection syndrome; SD standard deviation 


\section{Wexner and FIQoL Scale}

Walma et al. was the only study that reported on Wexner score and FIQoL. They reported that an ileostomy was an independent predictor for impaired FIQoL (OR -0.524, $95 \% \mathrm{CI},-1.072$ to $-0.021, p=0.041)$ and in the univariate analysis the Wexner score was negatively influenced by an ileostomy. Ileostomy reversal within 3 months after the index operation showed significantly better FIQoL (median 15, IQR 13.1-16 vs. 12, IQR 10.5-13.9; $p=0.001)$ and Wexner score (median of 5, IQR 2.5-8 vs. 10.5, IQR 6-13.8; $p<0.001$ ) than reversal after 3 months [20].

\section{MSKCC-BFI}

In the secondary analysis of the EASY trial, both the LARS score and the MSKCC-BFI score were used to assess bowel function in patients with early stoma closure within 2 weeks and after 16 weeks. Patients in the early closure group showed higher BFI scores compared to late closure group, which indicates better bowel control, although this was not statistically significant (median BFI score 71, IQR 59-75 vs. 63 IQR $60-70 p=0.27$ ) [11]. Participants in the late closure group did have a significantly worse BFI score on the urgency/soiling subscale (median score early group 14 vs late group $17, p=0.017$ ). A strong negative correlation was noted between the two scoring systems $(\rho=-0.72$, $p<0.001)$.

\section{Bowel function questionnaire by Hallbook}

Lindgren et al. used the bowel function questionnaire by Hallbook to assess anorectal function after LAR [21]. The presence of an ileostomy did not affect anorectal function, evaluated 1 year after stoma closure. However, no overall scores were presented, only scores of the different subscales. Median scores were the same in each group regarding need for medication, evacuation difficulties, fragmentation of bowel movements, incontinence and effects on wellbeing. On the urgency subscale patients with a stoma had a higher incidence of urgency compared to patients without a stoma, $35.5 \%$ vs. $25.4 \%$, although not statistically significant.

\section{Discussion}

In this systematic review and meta-analysis, we evaluated the impact of a defunctioning stoma, and time to stoma closure on bowel function after LAR for rectal cancer. The risk of developing major LARS was higher when a patient has had a defunctioning ileostomy compared to patients without an ileostomy. A prolonged time to ileostomy closure was associated with an increased incidence of major LARS.
The included studies were from Australia, China, the Netherlands, the United Kingdom, Spain and Sweden and therefore represent a wide geographical area. All included studies, with the exception of the study by Hughes et al. [1], have at least a follow-up time of 1 year. It has been demonstrated that bowel dysfunction improves over time, but the improvement is limited after one year, thus these results reliably represent bowel function outcome after LAR [21, 28].

A recent systematic review compared bowel function between patients undergoing LAR with and without ileostomy and outcomes for early versus later ileostomy closure [29]. Keane et al. included only four studies that assessed bowel function, and three of the four studies were from the same cohort of patients $[11,12,18,30]$. A pooled analysis was performed with two of the included studies which showed a twofold increase in the rate of major LARS in patients with an ileostomy (OR 1.96, 95\% CI, 1.1-3.5, $p=0.02$ ). This is in line with the results of our analysis, although through collection of raw data we were able to perform a pooled analysis that included six studies and showed an even stronger effect of an ileostomy on the risk of major LARS (OR 2.84, 95\% CI, 1.70-4.75, $p<0.0001$ ). Keane et al. report that they were unable to perform a meta-analysis on the influence of timing of ileostomy closure on bowel function.

The association between LARS and presence of an ileostomy could be due to a variety of reasons; an ileostomy is more likely to be created in low anastomoses and those patients are more likely to receive neoadjuvant therapy, which have both been shown to be associated with LARS [9]. The contribution of the length of the remnant rectum to post-operative function might be due to the interruption of the complex physiology of bowel motility and rectoanal coordination [19]. Suggested mechanisms for how radiotherapy impacts bowel function include nerve damage, impairment of the anal sphincter, and decreased neorectal compliance caused by radiation-induced fibrosis [15]. Nonetheless an ileostomy might have an independent influence on the LARS score. In the EASY trial (2019), the risk of major LARS was increased when there was a prolonged time to closure and results remained similar after adjusting for tumor height and use of radiotherapy [11] and several studies reported that both radiotherapy, tumor height as well as the presence of a defunctioning stoma were independent predictors of worse LARS score [15, 16].

Anastomotic leak has been mentioned as a risk factor for developing major LARS [4]. Patients who have an anastomotic leak will often have an ileostomy for a more prolonged period before closure. Of the included studies in our review, 5 assessed the association between an anastomotic leak and LARS where none of the studies confirmed a correlation [1, $12,15,17,22]$. In addition the prevalence of major LARS in almost $50 \%$ of the patients is much higher than the reported 
percentages of anastomotic leak, ranging from 0\%-12.7\% in the included studies. The low numbers of anastomotic leak might contribute to the lack of a significant impact on LARS score due to a type II error. Furthermore, anastomotic leak rates might be underreported in the presence of a covering stoma, when an asymptomatic leak might be present. Results from the Dutch SNAPSHOT study showed an increase in anastomotic leak within 30 days from $8.2 \%$ to $13.4 \%$, when retrospectively checking data from the registry, which increased to $20 \%$ at end of follow-up after occult leaks were found during stoma reversal [31].

Other findings pertaining to LARS include changes in the functional state of the pelvic floor and the sphincter complex during and after a LAR [32]. When not used for a prolonged period of time, in a defunctioned state, pelvic floor muscles are likely to lose strength and bowel function might be impaired as a result. This is in line with a systematic review by Visser et al. that demonstrated that the use of pelvic floor rehabilitation was useful for improving functional outcome after LAR [33].

Another reason for the association between an ileostomy and major LARS may be due to alterations in colonic environment and microbiota. There is loop ileostomy-mediated fecal stream diversion which alters microbiota composition, which in turn affects intestinal epithelial cell turnover and consequently impacts on intestinal structure and function [34]. One study comparing colonic flora of patient with and without an ileostomy found that the abundance of several types of bacteria differed significantly between the ileostomy and control groups [35]. This could be an explanation for the presence and duration of an ileostomy being an influence on the prevalence of major LARS.

Most surgeons aim to reverse a stoma within 2-4 months after initial surgery, but the scheduling of stoma closure is extremely variable among hospitals $[11,36]$. There are reports that it is safe to close a defunctioning stoma within 2 weeks after surgery, with a similar rate in post-operative complications and anastomotic leaks compared to delayed closure [37]. In the United Kingdom, the national bowel cancer audit reported that up to $40 \%$ of patients had a delay in closure of more than 18 months, which is confirmed in the CLOSE-IT trial [5]. None of the included studies reported on reason for delay in stoma closure. In previous reports, adjuvant chemotherapy is mentioned as a potential reason for delay, but when comparing patients who underwent closure of an ileostomy either during adjuvant chemotherapy or after chemotherapy a large Korean study found that postoperative hospital stay, post-operative complications, and disease free survival were equal in both groups [38]. While clinical factors might preclude timely closure, different healthcare systems with different reimbursement/funding and logistics will have a varying average in their time to stoma closure [39]. Currently there is a lack of guidance on the optimal timing of stoma closure.

There are some limitations to this systematic review. None of the included studies had a pre-study power calculation performed for this review's primary outcome variable. Within the randomized controlled studies, the assessment of bowel function after an ileostomy was part of a post-hoc analysis. The majority of the studies included were cohort studies without clear guidelines on when to defunction the anastomosis with a risk of selection bias. The decision to create an ileostomy is often left to the surgeon's discretion, and patients with an ileostomy might have a lower anastomosis, more complex surgery, and/or neoadjuvant therapy. This could explain the moderate heterogeneity between the included studies in our meta-analysis comparing LARS in patients with and without ileostomy. Furthermore, none of the studies reported on the effect of patient related factors such as body mass index and age on bowel function, nor was the influence of operative time addressed as indicator of the complexity of the surgery.

Finally there were five different tools used to assess bowel function though many more are described in the literature, which makes comparison between different studies difficult. The most commonly reported was the LARS score, a score specifically designed for rectal cancer patients after LAR [40]. However this score is thought to underestimate evacuatory dysfunction and may not accurately assess the impact of symptoms on individual patient's quality of life [41]. The interpretation of the LARS score results is also limited by the findings that major LARS is also common in the general population without rectal cancer, especially in women between 50 to 79 years old [42]. A new LARS score is being developed but so far optimal scoring systems to assess bowel function after LAR are lacking [41].

The cause of poor bowel function after rectal cancer surgery is multifactorial. Further studies assessing the pathophysiology of the association an ileostomy and major LARS and influence of timing on ileostomy closure are required with bowel function as primary outcome of the study.

\section{Conclusions}

Based on this meta-analysis, the risk of having major LARS seems higher with a defunctioning ileostomy, and prolonged time to ileostomy closure seem to have a negative effect on bowel function. Pros and cons of routine diversion during LAR should be reconsidered.

Supplementary Information The online version contains supplementary material available at https://doi.org/10.1007/s10151-021-02436-5. 
Author contributions I. V., R. H. and J. A. C. contributed to the study conception and design. Material preparation, data collection and analysis were performed by I. Vogel and N. Reeves. First draft was written by I. Vogel and all authors commented on previous versions of the manuscript. All authors have made substantial contributions to all aspects of the manuscript including the conception and design of the study, acquisition of data, analysis and interpretation of data, drafting the article or critical appraisal and revisions. All authors have read and approved the final version of the manuscript that is being submitted. The manuscript is currently not under consideration elsewhere.

\section{Declarations}

Conflicts of interest No funding was received for conducting this study. All authors certify that they have no affiliations with or involvement in any organization or entity with any financial interest or non-financial interest in the subject matter or materials discussed in this manuscript.

Open Access This article is licensed under a Creative Commons Attribution 4.0 International License, which permits use, sharing, adaptation, distribution and reproduction in any medium or format, as long as you give appropriate credit to the original author(s) and the source, provide a link to the Creative Commons licence, and indicate if changes were made. The images or other third party material in this article are included in the article's Creative Commons licence, unless indicated otherwise in a credit line to the material. If material is not included in the article's Creative Commons licence and your intended use is not permitted by statutory regulation or exceeds the permitted use, you will need to obtain permission directly from the copyright holder. To view a copy of this licence, visit http://creativecommons.org/licenses/by/4.0/.

\section{References}

1. Hughes DL, Cornish J, Morris C, LARRIS Trial Management Group (2017) Functional outcome following rectal surgerypredisposing factors for low anterior resection syndrome. Int $\mathbf{J}$ Colorectal Dis. 32(5):691-697

2. Montedori A, Cirocchi R, Farinella E, Sciannameo F, Abraha I (2010) Covering ileo- or colostomy in anterior resection for rectal carcinoma. Cochrane Database Syst Rev (5)

3. Hubbard G, Taylor C, Watson AJM, Munro J, Goodman W, Beeken RJ (2020) A physical activity intervention to improve the quality of life of patients with a stoma: a feasibility study. Pilot Feasibility Stud 6:12

4. Danielsen AK, Park J, Jansen JE, Bock D, Skullman S, Wedin A et al (2017) Early closure of a temporary ileostomy in patients with rectal cancer: a multicenter randomized controlled trial. Ann Surg 265(2):284-290

5. Vaughan-Shaw PG, Gash K, Adams K, Vallance AE, Pilkington SA, Torkington J et al (2018) Protocol for a multicentre, dual prospective and retrospective cohort study investigating timing of ileostomy closure after anterior resection for rectal cancer: The CLOSurE of Ileostomy Timing (CLOSE-IT) study. BMJ Open 8(10):e023305

6. Wood C, Lawton S, Downing A, Morris E, Glaser AW, Thomas J et al (2015) Quality of life of colorectal cancer survivors in England: report on a national survey of colorectal cancer survivors using Patient Reported Outcome Measures (PROMs)

7. Pieniowski EHA, Palmer GJ, Juul T, Lagergren P, Johar A, Emmertsen KJ et al (2019) Low anterior resection syndrome and quality of life after sphincter-sparing rectal cancer surgery: a longterm longitudinal follow-up. Dis Colon Rectum 62(1):14-20

8. Juul T, Battersby NJ, Christensen P, Janjua AZ, Branagan G, Laurberg $S$ et al (2015) Validation of the English translation of the low anterior resection syndrome score. Colorectal Dis 17(10):908-916

9. Croese AD, Lonie JM, Trollope AF, Vangaveti VN, Ho YH (2018) A meta-analysis of the prevalence of Low Anterior Resection Syndrome and systematic review of risk factors. Int J Surg $56: 234-241$

10. Moher D, Liberati A, Tetzlaff J, Altman DG (2010) Preferred reporting items for systematic reviews and meta-analyses: the PRISMA statement. Int J Surg 8(5):336-341

11. Keane C, Park J, Öberg S, Wedin A, Bock D, O'Grady G et al (2019) Functional outcomes from a randomized trial of early closure of temporary ileostomy after rectal excision for cancer. Br J Surg 106(5):645-652

12. Gadan S, Floodeen H, Lindgren R, Matthiessen P (2017) Does a defunctioning stoma impair anorectal function after low anterior resection of the rectum for cancer? A 12-year follow-up of a randomized multicenter trial. Dis Colon Rectum 60(8):800-806

13. Wells G, Shea B, O'Connell J (2014) The Newcastle-Ottawa Scale (NOS) for assessing the quality of nonrandomised studies in meta-analyses. Ottawa Health Research Institute Website 7

14. Jadad AR, Moore RA, Carroll D, Jenkinson C, Reynolds DJ, Gavaghan DJ et al (1996) Assessing the quality of reports of randomized clinical trials: is blinding necessary? Control Clin Trials 17(1):1-12

15. Sun W, Dou R, Chen J, Lai S, Zhang C, Ruan L et al (2019) Impact of long-course neoadjuvant radiation on postoperative low anterior resection syndrome and quality of life in rectal cancer: post hoc analysis of a randomized controlled trial. Ann Surg Ocol 26(3):746-755

16. van Heinsbergen M, Janssen-Heijnen ML, Leijtens JW, Slooter GD, Konsten JL (2018) Bowel dysfunction after sigmoid resection underestimated: Multicentre study on quality of life after surgery for carcinoma of the rectum and sigmoid. Eur J Surg Oncol 44(8):1261-1267

17. Jimenez-Gomez LM, Espin-Basany E, Trenti L, Martí-Gallostra M, Sánchez-García JL, Vallribera-Valls F, et al. (2017) Factors associated with low anterior resection syndrome after surgical treatment of rectal cancer. Colorectal Dis

18. Jiménez-Rodríguez RM, Segura-Sampedro JJ, RiveroBelenchón I, Díaz Pavón JM, García Cabrera AM, Vazquez Monchul JM et al (2017) Is the interval from surgery to ileostomy closure a risk factor for low anterior resection syndrome? Colorectal Dis 19(5):485-490

19. Bondeven P, Emmertsen KJ, Laurberg S, Pedersen BG (2015) Neoadjuvant therapy abolishes the functional benefits of a larger rectal remnant, as measured by magnetic resonance imaging after restorative rectal cancer surgery. Eur J Surg Oncol 41(11):1493-1499

20. Walma MS, Kornmann VNN, Boerma D, de Roos MAJ, van Westreenen HL (2015) Predictors of fecal incontinence and related quality of life after a total mesorectal excision with primary anastomosis for patients with rectal cancer. Ann Coloproctol 31(1):23-28

21. Lindgren R, Hallböök $O$, Rutegård J, Sjödahl R, Matthiessen $P$ (2011) Does a defunctioning stoma affect anorectal function after low rectal resection? Results of a randomized multicenter trial. Dis Colon Rectum 54(6):747-752

22. Sturiale A, Martellucci J, Zurli L, Vaccaro C, Brusciano L, Limongelli $P$ et al (2017) Long-term functional follow-up after anterior rectal resection for cancer. Int J Colorectal Dis $32(1): 83-88$ 
23. Emmertsen KJ, Laurberg S (2012) Low anterior resection syndrome score: development and validation of a symptom-based scoring system for bowel dysfunction after low anterior resection for rectal cancer. Ann Surg 255(5):922-928

24. Temple LK, Bacik J, Savatta SG, Gottesman L, Paty PB, Weiser MR et al (2005) The development of a validated instrument to evaluate bowel function after sphincter-preserving surgery for rectal cancer. Dis Colon Rectum 48(7):1353-1365

25. Vaizey CJ, Carapeti E, Cahill JA, Kamm MA (1999) Prospective comparison of faecal incontinence grading systems. Gut 44(1):77-80

26. Rockwood TH, Church JM, Fleshman JW, Kane RL, Mavrantonis C, Thorson AG et al (2000) Fecal incontinence quality of life scale: quality of life instrument for patients with fecal incontinence. Dis Colon Rectum 43(1):9-16; discussion 7

27. Hallbook O, Sjodahl R (2000) Surgical approaches to obtaining optimal bowel function. Semin Surg Oncol 18(3):249-258

28. Cheong C, Oh SY, Choi SJ, Suh KW (2019) Ultralow anterior resection and coloanal anastomosis for low-lying rectal cancer: an appraisal based on bowel function. Dig Surg 36(5):409-417

29. Keane C, Sharma P, Yuan L, Bissett I, O'Grady G (2019) Impact of temporary ileostomy on long-term quality of life and bowel function: a systematic review and meta-analysis. ANZ J Surg. 90(5):687-692

30. Floodeen H, Lindgren R, Hallbook O, Matthiessen P (2014) Evaluation of long-term anorectal function after low anterior resection: a 5-year follow-up of a randomized multicenter trial. Dis Colon Rectum 57(10):1162-1168

31. Borstlap WAAWE, Aukema TS, Bemelman WA, Tanis PJ (2017) anastomotic leakage and chronic presacral sinus formation after low anterior resection: results from a large cross-sectional study. Ann Surg 266(5):870-877

32. Ziv Y, Zbar A, Bar-Shavit Y, Igov I (2013) Low anterior resection syndrome (LARS): cause and effect and reconstructive considerations. Tech Coloproctol 17(2):151-162

33. Visser WS, Te Riele WW, Boerma D, van Ramshorst B, van Westreenen HL (2014) Pelvic floor rehabilitation to improve functional outcome after a low anterior resection: a systematic review. Ann Coloproctol 30(3):109-114
34. Beamish EL, Johnson J, Shaw EJ, Scott NA, Bhowmick A, Rigby RJ (2017) Loop ileostomy-mediated fecal stream diversion is associated with microbial dysbiosis. Gut Microbes 8(5):467-478

35. Baek SJ, Kim SH, Lee CK, Roh KH, Keum B, Kim CH et al (2014) Relationship between the severity of diversion colitis and the composition of colonic bacteria: a prospective study. Gut Liver 8(2):170-176

36. Moran B, Cunningham C, Singh T, Sagar P, Bradbury J, Geh I et al (2017) Association of Coloproctology of Great Britain \& Ireland (ACPGBI): guidelines for the management of cancer of the colon, rectum and anus (2017) - surgical management. Colorectal Dis 19(Suppl 1):18-36

37. Farag S, Rehman S, Sains P, Baig MK, Sajid MS (2017) Early vs delayed closure of loop defunctioning ileostomy in patients undergoing distal colorectal resections: an integrated systematic review and meta-analysis of published randomized controlled trials. Colorectal Dis 19(12):1050-1057

38. Choi YJ, Kwak JM, Ha N, Lee TH, Baek SJ, Kim J et al (2019) Clinical outcomes of ileostomy closure according to timing during adjuvant chemotherapy after rectal cancer surgery. Ann Coloproctol 35(4):187-193

39. Sier MF, van Gelder L, Ubbink DT, Bemelman WA, Oostenbroek RJ (2015) Factors affecting timing of closure and non-reversal of temporary ileostomies. Int J Colorectal Dis 30(9):1185-1192

40. Bryant CL, Lunniss PJ, Knowles CH, Thaha MA, Chan CL (2012) Anterior resection syndrome. Lancet Oncol 13(9):e403-e408

41. Keane C, Fearnhead NS, Bordeianou LG, Christensen P, Espin Basany E, Laurberg S et al (2020) International consensus definition of low anterior resection syndrome. ANZ J Surg 90(3):300-307

42. Juul T, Elfeki H, Christensen P, Laurberg S, Emmertsen KJ, Bager $P(2019)$ Normative data for the low anterior resection syndrome score (LARS Score). Ann Surg 269(6):1124-1128

Publisher's Note Springer Nature remains neutral with regard to jurisdictional claims in published maps and institutional affiliations. 